\title{
Optical Layer Modulation Format Conversion in OFDM-Based Flexible Optical Networks
}

\author{
Hejun $\mathrm{Zhu}^{1,2, *}$ and Liehuang $\mathrm{Zhu}^{3}$ \\ ${ }^{1}$ Computer College of Beijing Institute of Technology, Beijing 100081, P.R. China \\ ${ }^{2}$ Beijing Esafenet Network Security Technology Company Limited, Beijing 100085, P.R. China \\ ${ }^{3}$ Computer College of Beijing Institute of Technology, Beijing 100081, P.R. China \\ ${ }^{*}$ Corresponding author
}

\begin{abstract}
In this letter, we proposes and experimentally demonstrates an optical layer modulation format conversion technique from quadrature phase shift keying (QPSK) to 16 quadrature amplitude modulation (QAM) in orthogonal frequency division multiplexing (OFDM)-based flexible optical wavelength division multiplex (WDM) networks. The 16-QAMOFDM signal is generated by direct detection after the superposition of two QPSK-OFDM optical signals with different optical powers and wavelengths. The optical power ratio 2:1 of two QPSK-OFDM signals can be realized by adjusting the power of two coupled optical signal in switching nodes. The tolerance to symbol delay and power deviation of two QPSK-OFDM signals is also experimentally evaluated.
\end{abstract}

Keywords-orthogonal frequency division multiplexing; modulation format conversion; direct detection; flexible optical networks

\section{INTRODUCTION}

In a flexible optical WDM (FWDM) network, the fixed channel grid restrictions are removed and non-uniform and dynamic allocation of spectrum are allowed, leading to higher spectral efficiency compared to conventional fixed grid networks. A suitable technology for implementing FDWM networks is OFDM, which provides the ability to separate a single channel into multiple closely-allocated subcarriers with finer granularity and has better resistance to chromatic dispersion (CD) and polarization mode dispersion (PMD) [1]. The capacity of FWDM networks is usually limited by wavelength congestion at network nodes and system management requirements for network reconfiguration on link failure. Using wavelength converters and modulation format converters it is found that such restrictions can be relaxed by providing for more flexible spectrum allocation, thus allowing for evolution of the network. In the past, numbers of researches had demonstrated various conversion schemes in single carrier systems. Return to zero (RZ) could be converted to non-return to zero (NRZ) by using four-wave mixing (FWM) and dispersion in a delay-asymmetric nonlinear loop mirror [2]. Optical conversions techniques for M-ary PSK signals based on highly nonlinear fiber [3] or semiconductor optical amplifier [4] have also been proposed. The conversion from on-off keying (OOK) to binary phase-shift keying (RZ-BPSK) has been demonstrated utilizing cross-phase modulation (XPM) in a passive AlGaAs waveguide [5]. M-ary QAM signal generation schemes employing nonlinear optical loop mirror or highly nonlinear fiber have been also been realized in [6, 7]. However, to our knowledge, optical layer format converters from QSPK to 16 QAM in OFDM-based system have not been reported.

In this paper, for the first time, we propose and experimentally demonstrate an optical layer QPSK to 16 QAM format conversion technique in OFDM-based switching networks. Symbol delay of two converted signals is not allowed in single carrier system [8]. While to OFDM signal, the orthogonality of the converted OFDM signal is maintained for the use of cyclic prefix (CP). The symbol delay of two OFDM signals introduces special rotation of constellation, which could be recovered by training symbols. Finally, to verify the correctness of the principle, two 5 Gbaud QPSKOFDM signal after 40-km standard single-mode fibers (SSMF) transmission are converted to 16-QAM-OFDM signal, and the converted signal is successfully transferred another $40-\mathrm{km}$ SSMF. The system performance under symbol delay and the tolerance to power deviation of two QPSK-OFDM signals are evaluated in the following experiment. The results show that there is only $0.6 \mathrm{~dB}$ power penalty caused by the impact of symbol delay. Compared with symbol delay, the system performance is more robust to power deviation.

\section{PRINCIPLE}

Figure I (a) shows the configuration of the proposed modulation format converter. The two OFDM transmitters use a conventional intensity modulation scheme [9]. The output double-sideband (DSB) optical signals of two transmitters can be written as

$$
E_{1}=e^{j \omega_{1} t}\left(1+\sum_{n=1}^{N} v_{1, n} \cos \left(n \omega t+\theta_{1, n}\right)\right)
$$

and

$$
E_{2}=e^{j \omega_{2} t}\left(1+\sum_{n=1}^{N} v_{2, n} \cos \left(n \omega t+\theta_{2, n}\right)\right)
$$

where $\omega_{1}$ and $\omega_{2}$ are the angular frequency of optical carriers, $N$ is the subcarrier number, $v_{1, n}, v_{2, n}$ and $\theta_{1, n}, \theta_{2, n}$ are the amplitudes and phases of the $n^{\text {th }}$ subcarrier, and the second- 


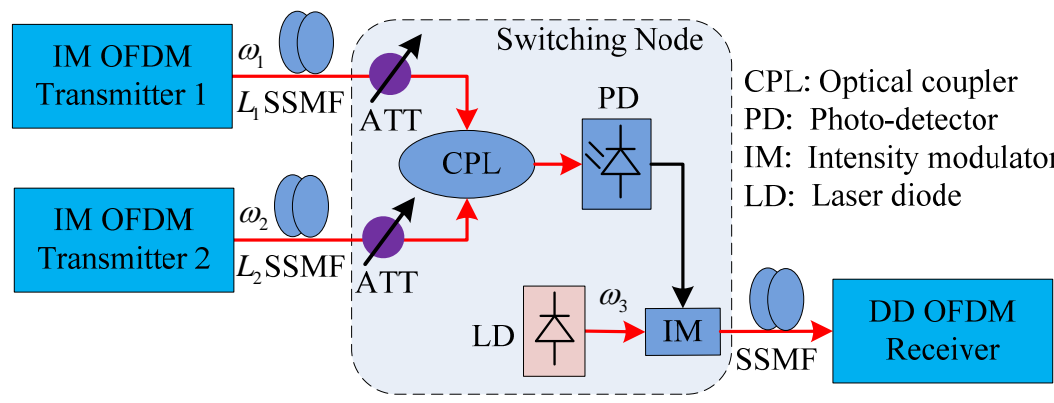

(a)

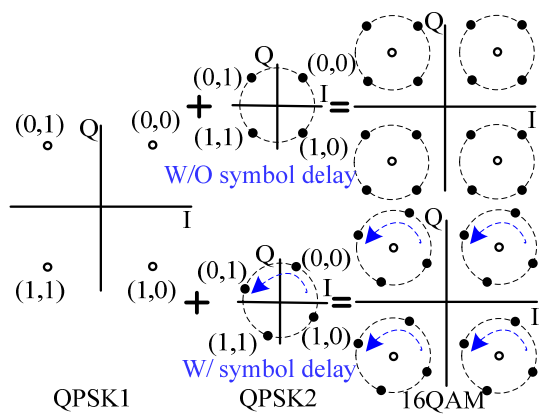

(b)

FIGURE I. (a)PRINCIPLE OF MODULATION FORMAT CONVERSION IN OFDM-BASED SWITCHING NODE. (b) THE 16 QAM CONSTELLATION CAN BE REPRESENTED AS THE COMBINATION OF TWO QPSK CONSTELLATIONS WITH AND WITHOUT SYMBOL DELAY.

and higher-order terms after power series expansion are disregarded [9]. After transmission with the distance of $\mathrm{L}_{1}$ and $\mathrm{L}_{2}$, the group-velocity dispersion parameter of $\beta_{2}$ and no fiber loss, then the optical signals become

$$
E_{1, C D}=e^{j \omega_{1} t}\left(1+\sum_{n=1}^{N} v_{1, n} e^{j\left(n^{2} \theta_{1, D}\right)} \cos \left(n \omega t+\theta_{1, n}\right)\right)
$$

and

$$
E_{2, C D}=e^{j \omega_{2} t}\left(1+\sum_{n=1}^{N} v_{2, n} e^{j\left(n^{2} \theta_{2, D}\right)} \cos \left(n \omega t+\theta_{2, n}\right)\right)
$$

where $\theta_{1, D}=\beta_{2} L_{1} \omega^{2} / 2$, and $\theta_{2, D}=\beta_{2} L_{2} \omega^{2} / 2$. The two optical signals at switching node are coupled and direct detected, then the received signal can be expressed as

$$
\begin{aligned}
R & =\left|E_{1, C D}\right|^{2}+\left|E_{2, C D}\right|^{2} \\
= & \sum_{n=1}^{N} v_{1, n} \cos \left(n^{2} \theta_{1, D}\right) \cos \left(n \omega t+\theta_{1, n}\right) \\
& +\sum_{n=1}^{N} v_{2, n} \cos \left(n^{2} \theta_{2, D}\right) \cos \left(n \omega t+\theta_{2, n}+n \theta_{\tau}\right) \\
= & \sum_{n=1}^{N}\left|r_{n}\right| \cos \left(n \omega t+\theta_{n}\right)
\end{aligned}
$$

where $n \theta_{\tau}$ represents the phase of symbol delay relative to $\mathrm{E}_{1, \mathrm{CD}}, \quad r_{n}=v_{1, n} \cos \left(n^{2} \theta_{1, D}\right) e^{j \theta_{1, n}}+v_{2, n} \cos \left(n^{2} \theta_{2, D}\right) e^{j\left(\theta_{2, n}+n \theta_{\tau}\right)}$ and $\theta_{n}$ is the compound angle of $r_{n} \cdot \cos \left(n^{2} \theta_{1, D}\right)$ and $\cos \left(n^{2} \theta_{2, D}\right)$ are the conventional formula of power fading [10]. The influence of power fading in short distance $(<40 \mathrm{~km})$ applications could be ignored [11], so $\cos \left(n^{2} \theta_{1, D}\right) \cong \cos \left(n^{2} \theta_{2, D}\right) \cong 1$.

To explain the principle of the converter, in Figure I (b), the 16 QAM constellations could be represented as the

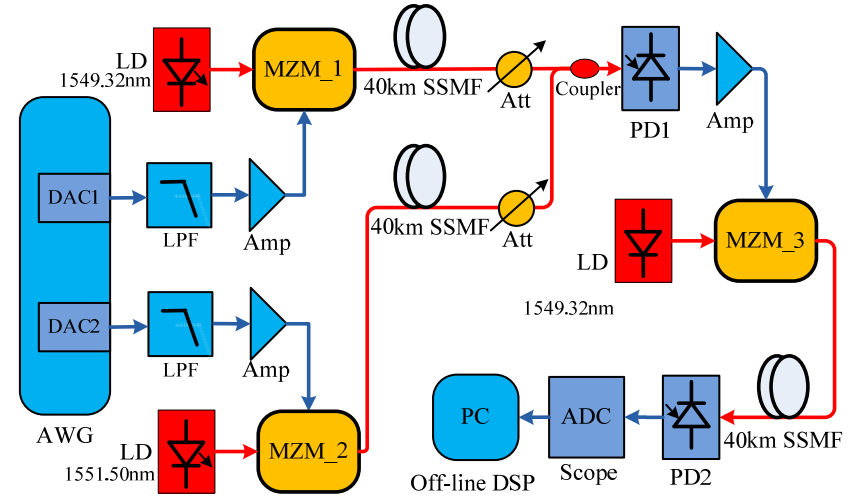

FIGURE II. EXPERIMENTAL SETUP OF QPSK TO 16 QAM FORMAT CONVERSION IN DIRECT DETECTION OFDM SYSTEM.

combination of two QPSK constellations. In order to achieve the constellation of 16 QAM signal, the amplitude of QPSK1 should be double that of QPSK2, $v_{1, n}: v_{2, n}=2: 1$. Each point of the original constellation is obtained as the product of a point from the first constellation (QPSK1) and a point from the second constellation (QPSK2). That is to say 16 QAM is generated by the superposition of these two QPSK signals. An ideal 16 QAM constellation will be produced when the two QPSK-OFDM signals are perfectly synchronized, the constellation of top-right corner shown in Figure I (b). However, in the actual network, signals from different transmitters are difficult to achieve absolute symbol synchronization, which is caused by the phase noise of clock recovery circuit [13] and symbol timing error. So a special rotation of 16 QAM constellation is happened, the constellation of bottom right corner shown in Figure I (b). For each subcarrier, the ideal rotated 16 QAM constellation could be obtained by using training symbols. The demodulation of data subcarrier is realized by the calculation and comparison of the minimum Euclidean distance between the ideal rotated constellation and the signal after equalization. 


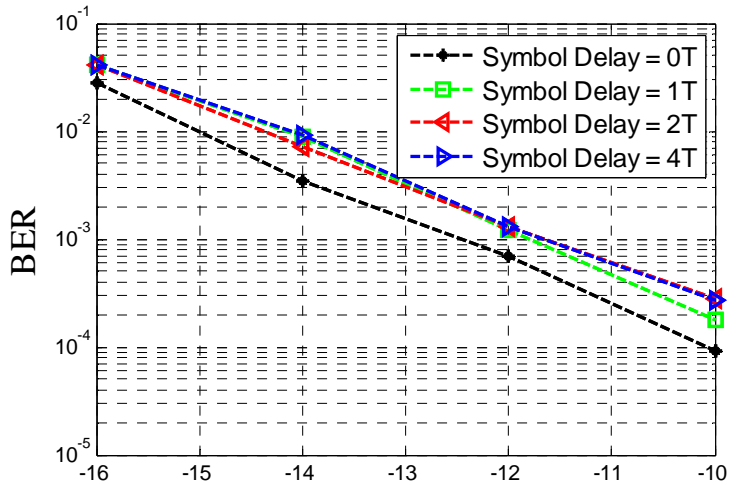

(a) Received Power (dBm)

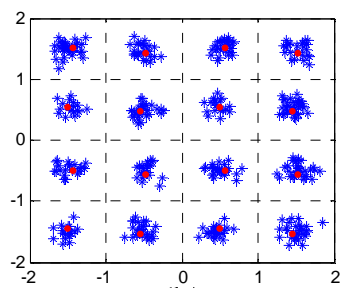

(b)

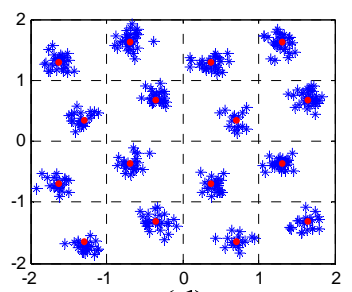

(d)

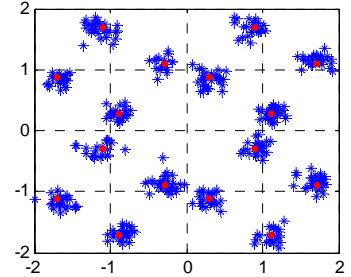

(c)

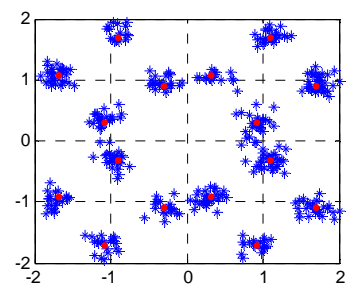

(e)
FIGURE III. (a)BER VERSUS THE RECEIVED POWER OF PD2 AT DIFFERENT SYMBOL DELAY. THE CONSTELLATION AFTER EQUALIZATION AT SUBCARRIER NUMBER OF (b) $\mathrm{N}=1$, (c) $\mathrm{N}=25$, (d) $\mathrm{N}=50$, AND (e) $\mathrm{N}=100$.

\section{EXPERIMENTAL SETUP AND RESULTS}

The experimental setup is shown in Figure II. Two channel baseband electrical OFDM signals are generated by an arbitrary waveform generator (AWG, Tektronix ${ }^{\circledR}$ AWG7122) using the MATLAB ${ }^{\circledR}$ program. The sampling rate and digital to analog converter (DAC) resolution of AWG are 12 GSample/s and 8 bits, respectively. The OFDM signal contains 107 subcarriers of QPSK format to occupy $5-\mathrm{GHz}$ bandwidth with an inverse fast Fourier transform (IFFT) size of 256, yielding a total raw data rate of 10 Gbps for each channel. CP of $1 / 8$ is used. A low pass filter (LPF) with $3-\mathrm{dB}$ bandwidth of $5.5 \mathrm{GHz}$ is inserted after AWG to prevent highfrequency residual images. The electrical signal after microwave amplifier is used to drive a single drive MachZehnder modulator (MZM), which is biased at its quadrature point to generate an optical DSB signal. The optical powers after 40-km SSMF transmission and without the use of erbium doped fiber amplifier (EDFA) are adjusted by two optical attenuators. 16 QAM signal could be obtained after opticalelectrical $(\mathrm{O} / \mathrm{E})$ conversion of the coupled optical signal. The

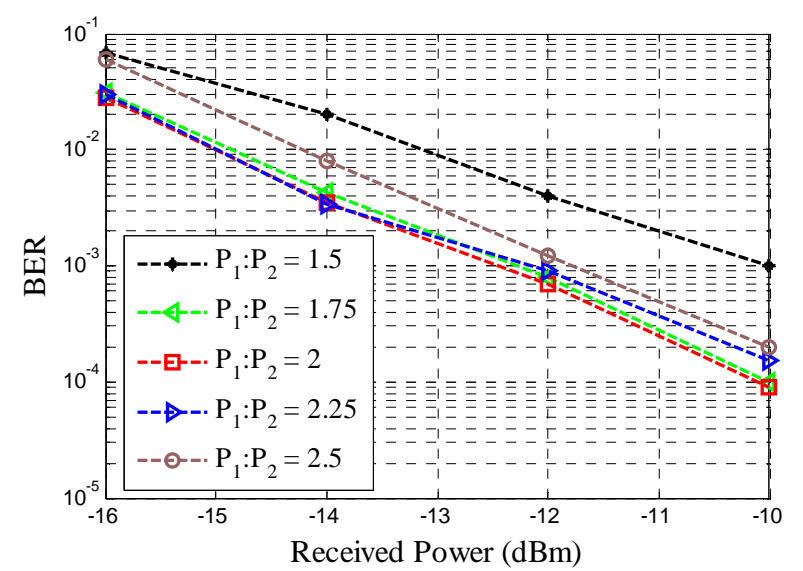

FIGURE IV. BER VERSUS THE RECEIVED POWER WITH DIFFERENT POWER DEVIATION.

16 QAM-OFDM electrical signal after the third microwave amplifier is used to drive the third MZM with the same configuration as the first two. The launch powers and optical modulation index (OMI) of the three transmitters are all set to be $6 \mathrm{dBm}$ and 0.25 . The received electrical signal after another 40-km SSMF transmission and O/E conversion is captured by a LeCroy ${ }^{\circledR}$ digital oscilloscope (WaveMaster813ZI-A) with 40-GSample/s sampling rate and 8-bit resolution. The received signal is processed off-line by MATLAB ${ }^{\circledR}$ digital signal processing (DSP) program. The OFDM signal after direct detection will suffer from subcarrier-to-subcarrier intermixing interference (SSII) [9], [11, 12], which will reduce the accuracy of channel estimation and the reconstruction of ideal rotated constellation. Specially designed training symbols with subcarrier interleaving [14] and lower peak to average power ratio (PAPR) are used to protect the training symbols from being affected by SSII.

To give an analysis of the influence of symbol delay on system performance, the two QPSK-OFDM signals at B2B are pre-emphasis [15] before IFFT to get a flat signal-to-noise ratio (SNR) curves for all subcarriers. The amplitude ratio of two QPSK signals is fixed to $v_{1, n}: v_{2, n}=2: 1$ and the received power of the PD1 is fixed to $-3 \mathrm{dBm}$. Bit error rate (BER) versus the received power at different symbol delay after 80km SSMF transmission are shown in Figure III (a). Compared with zero symbol delay case, there are only $0.6 \mathrm{~dB}$ power penalty at BER of $1 \times 10^{-3}$ (the FEC limit) with symbol delay = $1 \mathrm{~T}, 2 \mathrm{~T}$ and $4 \mathrm{~T}$, where $\mathrm{T}=1 / 12 \times 10^{9}$ (seconds) is the sampling interval of DAC. The SNR of each subcarrier has not changed for different symbol delay cases at the same received power. The reason of power penalty is that special rotation of 16 QAM constellation decreases the Euclidean distance. The constellations of data symbols and training symbols, after equalization, with received power of $-3 \mathrm{dBm}$ and symbol delay of $1 \mathrm{~T}$, at different subcarrier number of $n=1,25,50$ and 100 are shown by blue points and red points in Figure III (b-e). The corresponding SNR of data symbols are $20.31 \mathrm{~dB}, 20.25$ $\mathrm{dB}, 20.10 \mathrm{~dB}$, and $18.90 \mathrm{~dB}$. The decline of signal quality at higher frequency subcarrier is attributed to the power fading and SSII [9], [11, 12]. 
Figure IV shows the BER versus the received power of PD2 after $80-\mathrm{km}$ SSMF transmission with different power deviation of two QPSK-OFDM optical signals. The symbol delay of two coupled optical signal is set to be zero by adjusting the time delay of AWG output signal and the received power of PD1 is fixed to $-3 \mathrm{dBm}$. The three curves with power ratio of $\mathrm{P}_{1}: \mathrm{P}_{2}=1.75,2$, and 2.25 are overlapped. Moreover, compared with symbol delay of two QPSK signals, the system performance is more robust to power deviation of two coupled optical signals.

\section{CONCLUSIONS}

In this paper, we propose and experimentally demonstrate a QPSK signal to 16 QAM format conversion method in direct detection optical OFDM FWDM networks. The feasibility of the proposed method is confirmed by experiments. The impacts of symbol delay and power deviation of the two coupled optical signals on system performance are evaluated.

\section{REFERENCES}

[1] P. N. Ji, A. N. Patel, D. Qian, J. P. Jue, J. Hu, Y. Aono, and T. Wang, "Optical Layer Traffic Grooming in Flexible Optical WDM (FWDM) Networks," in Proc. ECOC, Geneva, 2011, paper We.10.P1.102.

[2] L. Wang, Y. Dai, G. K. P. Lei, J. Du, and C. Shu, "All-Optical RZ-toNRZ and NRZ-to-PRZ Format Conversions Based on DelayAsymmetric Nonlinear Loop Mirror,” IEEE Photon. Technol. Lett., vol. 23, no. 6, pp. 368-370, 2011.

[3] G. W. Lu, E. Tipsuwannakul, C. Lundström, M. Karlsson, and P. A. Andrekson, "Format Conversion From 120-Gb/s RZ-D8PSK to 80-Gb/s RZ-DQPSK Through FWM-Based Optical Phase Erasure,” IEEE Photon. Technol. Lett., vol. 22, no. 24, pp. 1817-1819, 2010.

[4] B. Zou, Y. Yu, X. Huang, Z. Wu, W. Wu, and X. Zhang, "All-Optical Format Conversion for Multichannel QPSK Signals," J. Lightwave Technol. , vol. 31, no. 3, pp. 375-384, 2013.

[5] W. Astar, P. Apiratikul, B. M. Cannon, T. Mahmood, J. J. Wathen, J. V. Hryniewicz, S. Kanakaraju, C. J. K. Richardson, T. E. Murphy, and G. M. Carter, "Conversion of RZ-OOK to RZ-BPSK by XPM in a Passive AlGaAs Waveguide,” IEEE Photon. Technol. Lett., vol. 23, no. 19, pp. 1397-1399, 2011.

[6] G. Huang, Y. Miyoshi, A. Maruta, Y. Yoshida, and K. I. Kitayama, “AllOptical OOK to 16-QAM Modulation Format Conversion Employing Nonlinear Optical Loop Mirror,” J. Lightwave Technol., vol. 30, no. 9, pp. 1342-1350, 2012.

[7] B. Zhang, H. Zhang, C. Yu, X. Cheng, Y. Kee Yeo, P. K. Kam, J. Yang, H. Zhang, Y. H. Wen, and K. M. Feng, "Conversion of RZ-OOK to RZBPSK by XPM in a Passive AlGaAs Waveguide," IEEE Photon. Technol. Lett., vol. 25, no. 4, pp. 327-330, 2013.

[8] M. Asghari, I. H. White, and R. V. Penty, "Wavelength Conversion Using Semiconductor Optical Amplifiers,” J. Lightwave Technol., vol. 15, no. 7, pp., 1181-1190, 1997.

[9] C. Wei, "Small-signal analysis of OOFDM signal transmission with directly modulated laser and direct detection,” Opt. Lett., vol. 36, no. 2, pp. 151-153, 2011.

[10] U. Gliese, S. Ngrskov, and T. N. Nielsen, "Chromatic Dispersion in Fiber-Optic Microwave and Millimeter-Wave Links,” IEEE Trans. Microwave Theory Tech., vol. 44, no. 10, pp. 1716 -1724, 1996.

[11] C. Ju, X. Chen, and Z. Zhang, “40Gbps 100-km SSMF VSB-IMDD OFDM Transmission Experiment Based on SSII Cancellation and FBGFiltering," in Proc. OFC/NFOEC, San Francisco, 2014, paper Tu2G.6.

[12] C. Ju, X. Chen, N. Liu, and L. Q. Wang, "SSII Cancellation in 40Gbps VSB-IMDD OFDM System Based on Symbol Pre-distortion," in Proc. ECOC 2014, Paper P.7.9.

[13] Q. Wang, L. Huo, Y. Xing, C. Lou, and B. Zhou "Low-Phase-Noise Clock Recovery from NRZ Signal and Simultaneous NRZ-to-RZ Format
Conversion,” IEEE Photon. Technol. Lett., vol. 25, no. 23, pp. 23392341, 2013.

[14] W. R. Peng, B. Zhang, K. M. Feng, X. Wu, A. E. Willner, and S. Chi, "Spectrally efficient direct-detected OFDM transmission incorporating a tunable frequency gap and an iterative detection techniques," $J$. Lightwave Technol., vol. 27, no. 24, pp. 5723-5735, 2009.

[15] Y. Gao, J. Yu, J. Xiao, Z. Cao, F. Li, L. Chen, "Direct-Detection Optical OFDM Transmission System With Pre-Emphasis Technique," $J$. Lightwave Technol., vol. 29, no. 14, pp. 2138-2145, 2011. 